5. Which of the following statements about perforating corneal trauma cases is incorrect?

a. Can be caused by a cat claw injury

b. Are at risk of endophthalmitis c. May also have lens capsule tear caused by the original injury

d. Always require enucleation

\section{Managing corneal sequestra in cats}

\section{David Maggs}

Corneal sequestration is a disease that is unique to the cat. It is characterised by gradual progression of an amber, brown or black discoloration of usually the central cornea. Histologically it is described as a region of necrotic stroma that produces a 'foreign body-type' response with perilesional corneal blood vessels (and sometimes granulation tissue) and variably intense corneal oedema and/or inflammatory cell infiltration. Prior and usually chronic ulceration is common but not always reported. Sequestra are usually unilateral, but may occur bilaterally. Frequently, the eye appears painful, but some cats display only minor signs of discomfort. The unusual appearance is considered diagnostic, however differential diagnoses should include corneal foreign body, anterior synechiation/staphyloma or limbal melanoma. A breed predilection for Persian, Himalayan and Siamese cats exists. Recent evidence suggests that feline herpesvirus (FHV-1) may be involved in approximately 50\% of cases. However, other causes of chronic corneal irritation such as entropion, distichiasis, tear film deficiencies and lagophthalmos appear to also predispose to sequestrum formation. Identification and correction of any underlying causes are important where possible.

Although there is some difference of opinion about medical versus surgical treatment of these lesions, in the author's opinion lamellar keratectomy is the treatment of choice, especially if the cat is in pain or the lesion is deep or chronic. Placement of a corneal or conjunctival graft may be necessary if the keratectomy is deep. If the cat appears comfortable, medical management may be attempted as sequestra may spontaneously slough over a period of weeks to months. However, perforation during this natural progression of disease is possible and the degree of discomfort often makes this an untenable option. Medical management includes use of prophylactic topical antibiotics, antiviral medications and topical tear supplements that are mucinomimetic. The author prefers hyaluronate-containing artificial tears. Reflex uveitis should be treated; however cycloplegic agents should be used at minimum required doses since they decrease tear production, and neither topical corticosteroids nor non-steroidal anti-inflammatory drugs (NSAIDs) should be used due to concurrent ulceration and the risk of herpetic involvement. While systemically administered corticosteroids should definitely be avoided if herpetic reactivation is a concern, the possibility of systemically administered NSAIDs to also do this is less clear. Surgical procedures to reduce corneal exposure and irritation such as temporary or permanent partial tarsorrhaphy and correction of entropion should be considered in select cases. Recurrence is unfortunately relatively common in predisposed individuals and is reported to occur in as many as 33\% of cats. Complete excision and possibly graft placement may limit recurrence according to some studies. Surgical procedures such as temporary or permanent partial tarsorrhaphy and correction of entropion to reduce corneal exposure and irritation should be considered in selected cases.

\section{KEY LEARNING OBJECTIVES}

- To recognise the characteristic appearance and likely pathogenesis of corneal sequestration in cats

- To understand and balance the advantages and disadvantages of surgical versus medical therapy of this disease

- To formulate a complete and rationalised medical treatment plan for a cat with a corneal sequestrum

\section{MULTIPLE CHOICE QUESTIONS}

1. Which of the following statements about corneal sequestra in cats is true?
a. Are typically bilateral
b. Occur most often behind the third eyelid
c. Are associated with feline herpesvirus infection and other causes of chronic irritation
d. Produce an autoimmune reaction in the cornea

2. Which of the following statements about corneal sequestra in cats is true?
a. Require surgical excision with histopathology
b. Show a breed predilection for Scottish Folds
c. Recur in about $33 \%$ of cats
d. Are expensive and not easily handled in a general practice

3. Which of the following statements about medical therapy for corneal sequestra in cats is true?
a. Typically includes a topical antibiotic
b. Does not require tear supplementation
c. Should include a topical or systemic steroid
d. Requires that a topical NSAID is used to control the secondary uveitis

4. What may corneal sequestra be caused by?
a. Entropion
b. Lagophthalmos
c. Feline herpesvirus
d. All of the above

5. Which of the following statements about medical management of corneal sequestra is true?
a. May be associated with perforation
b. Should include a topical steroid
c. Is preferred over surgical resection
d. Need not be considered if surgery is performed 